\title{
Neuroprotective Effects of Endogenous Secretory Receptor for Advanced Glycation End-products in Brain Ischemia
}

\author{
Yu Shimizu ${ }^{1,2}$, Ai Harashima ${ }^{1}$, Seiichi Munesue ${ }^{1}$, Masahiro Oishi ${ }^{1,2}$, Tsuyoshi Hattori ${ }^{3}$, Osamu \\ Hori $^{3}$, Yasuko Kitao ${ }^{3}$, Hiroshi Yamamoto ${ }^{1,4}$, Nontaphat Leerach ${ }^{1}$, Mitsutoshi Nakada ${ }^{2}$, Yasuhiko \\ Yamamoto $^{1, *}$, Yasuhiko Hayashi ${ }^{2,5}$ \\ ${ }^{1}$ Department of Biochemistry and Molecular Vascular Biology, ${ }^{2}$ Department of Neurosurgery and ${ }^{3}$ Department \\ of Neuroanatomy, Kanazawa University Graduate School of Medical Sciences, Kanazawa 920-8641, Japan. \\ ${ }^{4}$ Komatsu University, Komatsu, Ishikawa 923-8511, Japan. \\ ${ }^{5}$ Department of Neurosurgery, Kanazawa Medical University, Uchinada 920-0293, Japan.
}

[Received January 21, 2019; Revised June 21, 2019; Accepted July 15, 2019]

\begin{abstract}
The receptor for advanced glycation end-products (RAGE) is expressed on human brain endothelial cells (HBEC) and is implicated in neuronal cell death after ischemia. We report that endogenous secretory RAGE (esRAGE) is a splicing variant form of RAGE that functions as a decoy against ischemia-induced neuronal cell damage. This study demonstrated that esRAGE was associated with heparan sulphate proteoglycans on HBEC. The parabiotic experiments between human esRAGE overexpressing transgenic (Tg), RAGE knockout (KO), and wild-type (WT) mice revealed a significant neuronal cell damage in the CA1 region of the WT side of parabiotic WT $\rightarrow$ WT mice, but not of $\mathrm{Tg} \rightarrow$ WT mice, 7 days after bilateral common carotid artery occlusion. Human esRAGE was detected around the CA1 neurons in the WT side of the parabiotic $\mathrm{Tg} \rightarrow$ WT pair, but not in the KO side of the $\mathrm{Tg} \rightarrow \mathrm{KO}$ pair. To elucidate the dynamic transfer of esRAGE into the brain, we used the blood-brain barrier (BBB) system (PharmaCo-Cell) with or without RAGE knockdown in endothelial cells. A RAGE-dependent transfer of esRAGE was demonstrated from the vascular to the brain side. These findings suggested that esRAGE is associated with heparan sulphate proteoglycans and is transferred into the brain via BBB to exert its neuroprotective effects in ischemia.
\end{abstract}

Key words: Receptor for advanced glycation end-products (RAGE), endogenous secretory RAGE (esRAGE), parabiosis, delayed neuronal cell damage, blood-brain barrier

Cerebral ischemic injury is one of the main causes of death and disability. Ischemic stroke, which results in insufficient supply of glucose and oxygen to brain tissues, could cause significant neuronal damage leading to neuronal cell death $[1,2]$. Transient global cerebral ischemia induces delayed neuronal death in the hippocampal CA1 subfield [3,4]. The hippocampus is not only responsible for many central nervous system functions including cognition, learning, and memory but is also one of the most vulnerable brain regions to various neurological insults such as hypoxia-ischemia, seizure, and prolonged stress [5-8]. The CA1 subfield has low ischemic tolerance, similarly to the striatum, thalamic reticular nucleus, and cerebellar Purkinje cells [9-11].

*Correspondence should be addressed to: Dr. Yasuhiko Yamamoto, Department of Biochemistry and Molecular Vascular Biology, Kanazawa University Graduate School of Medical Sciences, Kanazawa 920-8640, Japan. E-mail: yasuyama@med.kanazawa-u.ac.jp

Copyright: (C) 2019 Shimizu Y et al. This is an open-access article distributed under the terms of the Creative Commons Attribution License, which permits unrestricted use, distribution, and reproduction in any medium, provided the original author and source are credited. 
There are many hypotheses about the molecular mechanisms underlying the delayed neuronal cell death in ischemia, including mitochondrial dysfunction, oxidative stress, nitrosative stress and the calcium-glutamine hypothesis [12-15].

The receptor for advanced glycation end-products (RAGE) is a pattern-recognition receptor that is involved in ischemic brain injury [16-18]. It is also well known that RAGE is implicated in the development of diabetic vascular complications, atherosclerosis, neurodegenerative diseases, and inflammatory diseases [1924]. In the brain, RAGE expression is reported in microglial and endothelial cells $[12,25]$. Kamide et al. demonstrated that the expression of RAGE could rise in the cerebrovascular endothelium and hippocampal neurons after transient brain ischemia by bilateral common carotid artery occlusion (BCCAO) [16]. We have previously identified and reported endogenous soluble RAGE (esRAGE), which is a truncated variant of RAGE isoforms generated by alternative splicing of the pre-mRNA transcripts and detectable in human circulation [26]. Recombinant proteins of the soluble form of RAGE were generated with gene technology and used in animal experiments [27, 28]. Soluble forms of RAGE proteins are known to be generated in vivo by cleavage of membrane-bound full-length RAGE as well as esRAGE by metalloproteinsases [22]. The soluble RAGE proteins including esRAGE and the cleaved forms of RAGE are known to act as a decoy receptor and to ameliorate pathologies in experimental animal models [29]. For example, the application of soluble RAGE significantly reduced the infarct size and showed neuroprotective effects on cortical neurons in mouse MCAO models [30, 31]. In addition, the expression of inflammatory molecules such as tumor necrosis factor $\alpha$ or inducible nitric oxide synthase was attenuated at the mRNA level in the hippocampal CA1 region of esRAGE transgenic (Tg) mice and in RAGE knockout (KO) mice, when compared with wild-type (WT) mice [16]. The neuroprotective role of esRAGE was observed. Regarding the esRAGE Tg mice used in the report, the mice were created to produce human esRAGE in the liver using the albumin promoter [28]. However, human esRAGE was also detected in the brain of this mouse model. Therefore, to evaluate the strict effect of only bloodstream esRAGE, we employed the parabiotic strategy [32], which will demonstrate a stable level of blood esRAGE, as a pilot study of intravenous injection of esRAGE protein.

In the present study, we found that blood esRAGE significantly inhibited transient brain ischemia-induced neuronal cell damage and apoptosis in the hippocampal CA1 subfield in the mouse BCCAO model. Blood esRAGE was transferred into the brain through the bloodbrain barrier (BBB) by RAGE on endothelial cells, potentially contributing to the neuroprotective effects in ischemia. Thus, endothelial RAGE has two different roles in ischemia, namely as an inducer of vascular injury and neuronal damage and as a transporter of esRAGE, a neuroprotector, to the brain.

\section{MATERIAL AND METHODS}

\section{Animals}

All animal experiments were conducted according to the Guidelines for Proper Conduct of Animal Experiments by the Science Council of Japan (1 June 2006) (www.scj.go.jp/ja/info/kohyo/pdf/kohyo-20-k16-2e.pdf). All animal procedures were performed in accordance with and approved by the Animal Care and Use Committee of Kanazawa University (AP-132929). The report was written in compliance with the ARRIVE guidelines (Animal Research: Reporting in Vivo Experiments) (www.nc3rs.org.uk/arrive-guidelines). The RAGE KO $\left(\right.$ Ager $\left.^{-1}\right)$ and WT (C57BL/6J) mice were produced by crossbreeding heterozygous mutant mice [20]. The esRAGE $\mathrm{Tg}$ (C57BL/6J) mice were generated as described previously [28]. Female mice of each cohort (age, 8-12 weeks; weight, 20-30 g) were used in the experiments. General anesthesia was induced with $2.0 \%$ sevoflurane and was maintained with $0.5 \%$ sevoflurane using a face mask [16]. To introduce parabiosis, mirror image incisions were made through the skin at the left and right flanks. The skin of the adjacent parabiont was sutured together. Before surgery and $14 \mathrm{~d}$ postoperatively, venous blood samples $(100 \mu \mathrm{l})$ were collected from the tail vein. esRAGE levels in sera, culture media and tissue lysates were measured by human esRAGE enzyme-linked immunosorbent assay (ELISA) kit (BBridge) [28]. Cross-circulation was confirmed in a subset of parabiotic pairs by measuring the esRAGE blood concentration from one partner in the blood of the other partner [32]. Serum mouse soluble RAGE (sRAGE) was assayed with a mouse RAGE Quantikine ELISA kit (MRG00, R\&D Systems, Inc., MN, USA). Protein concentrations of tissue lysate were measured using a BCA protein assay kit (Pierce Biotechnology, Rockford, IL, USA).

\section{Surgical procedures (BCCAO)}

Seven days after parabiosis, brain ischemia was induced by BCCAO for 20 min with the use of microvascular clips as described previously [16]. Laser-Doppler flowmetry was used to measure the cerebral cortical microperfusion (3 mm lateral to bregma). In our experimental model, mice that demonstrated $<15 \%$ of baseline control microperfusion during the first minute of occlusion were 
used for subsequent experiments. Rectal temperature was maintained at $36.5-37.5^{\circ} \mathrm{C}$ by using a heat lamp and a blanket [16].

\section{Cell culture and immunofluorescence study}

Human microvascular endothelial cells (HMVEC) isolated from the neonatal dermis were maintained in HuMedia-EB2 medium supplemented with HumediaMvG (KURABO KE6550) containing 5\% (v/v) fetal bovine serum (FBS), $5 \mathrm{ng} / \mathrm{ml}$ basic fibroblast growth factor (bFGF), $10 \mu \mathrm{g} / \mathrm{ml}$ heparin, $10 \mathrm{ng} / \mathrm{ml}$ epidermal growth factor (EGF), $1 \mu \mathrm{g} / \mathrm{ml}$ hydrocortisone, and 39.3 $\mu \mathrm{g} / \mathrm{ml}$ dibutyryl cAMP, according to the manufacturer's instructions (Kurabo). Primary human brain microvascular endothelial cells (HBEC) were cultured with the CSC complete Recombinant Medium (Cell Systems Corporation) and cultivated in a humidified 37 ${ }^{\circ} \mathrm{C}$ incubator with $95 \%$ room air and 5\% $\mathrm{CO} 2$. Experiments were performed in an 8-well chamber slide (LAB-TEK $^{\mathrm{TM}}$ CHAMBER SLIDE ${ }^{\mathrm{TM}}$ SYSTEM, 177445JP). Recombinant human esRAGE protein produced previously in our lab was used for the experiments [26, 28, 33]. Briefly, esRAGE $(1 \mu \mathrm{g} / \mathrm{ml})$ was added to the culture media and incubated for $1 \mathrm{~h}$ with or without treatment with heparin $(0.1 \mathrm{IU} / \mathrm{ml})$ or heparitinase (1 mU/ml). After washing, cells were fixed with $4 \%$ paraformaldehyde for $30 \mathrm{~min}$, and the esRAGE signal was detected with the primary polyclonal anti-esRAGE antibody (1:200) in combination with the secondary antibody Alexa 488-conjugated goat anti-rabbit (Alexa Fluor ${ }^{\circledR} 488$ Goat Anti-rabbit IgG [H+L]; 1:500; Molecular Probes®) [25, 33, 34, 35]. For the detection of heparin sulfate, we used a monoclonal anti-heparan sulfate (10E4) antibody (Seikagaku Corporation, Japan) in combination with Alexa 647-comjugated goat antimouse IgM antibody (ab150123, Abcam). Coverslips were mounted onto glass slides using mounting medium (UltraCruz $^{\mathrm{TM}}$ Mounting Medium, Santa Cruz Biotechnology) [28]. Images were acquired by using wide-field fluorescence microscopes (EVOS ${ }^{\circledR}$ FL, Advanced Microscopy Group or BZ-9000, Keyence Co.)

\section{Immunohistochemistry}

Following transcardial perfusion with $4 \%$ paraformaldehyde, brains were dissected and post-fixed in $4 \%$ paraformaldehyde for $24 \mathrm{~h}$ at $4{ }^{\circ} \mathrm{C}$. Coronal sections (thickness $5 \mu \mathrm{m}$ ) of paraffin-embedded specimens were cut on a cryostat. Deparaffinization was performed by incubating the slides in the following solutions: (1) xylene bath (twice for $5 \mathrm{~min}$ each), (2) 100\% ethanol (twice for 3 min each), (3) $90 \%$ ethanol, (4) $80 \%$ ethanol, and (5) double distilled water [4]. Subsequently, the slides were heated in $6 \mathrm{mM}$ sodium citrate buffer $(\mathrm{pH} \mathrm{6.0)}$ at 95-100 ${ }^{\circ} \mathrm{C}$ for $10 \mathrm{~min}$ by using a microwave, and incubated at room temperature for $30 \mathrm{~min}$ in phosphate-buffered saline (PBS) and $1 \%$ bovine serum albumin (BSA), followed by anti-esRAGE antibody in combination with Alexa 488conjugated goat anti-rabbit (Alexa Fluor ${ }^{\circledR} 488$ Goat antirabbit IgG $[\mathrm{H}+\mathrm{L}]$ antibody, 1:500, Molecular Probes $\left.{ }^{\circledR}\right)$ in PBS $1 \%$ BSA [25]. Images were obtained by using a widefield fluorescence microscope (EVOS ${ }^{\circledR}$ FL, Advanced Microscopy Group). For co-staining for neuronal or astrocyte marker and human esRAGE, mouse brains were removed after perfusion with $4 \%$ paraformaldehyde, and coronal sections $(20-\mu \mathrm{m}$-thick sections) were cut on a cryostat (CM1950, Leica Biosystems, Wetzler, Germany) after cryoprotection using 30\% sucrose. Sections were processed for immunostaining with antibodies against NeuN (MAB377, Millipore, Billerica, MA), GFAP (G3893, Sigma, St. Louis, MO) and human esRAGE [26]. Alexa488 (Invitrogen, Carlsbad, CA)- conjugated donkey anti-mouse or Cy3-conjugated anti-rabbit secondary antibody (Jackson ImmunoResearch Laboratories, West Grove, PA) were used for visualization of immunolabeling. Imaging was performed on a laser scanning confocal microscope (Eclipse TE2000U, Nikon, Tokyo, Japan) with the Nikon EZ-C1 software.

\section{Neuronal cell quantification}

Hippocampal cell death was assessed according to a standard protocol described previously [36, 37]. Brain sections were stained with cresyl violet (Nissl) staining and hematoxylin-eosin staining. Cell counting was performed in five sections per hippocampus starting at 1.4 $\mathrm{mm}$ posterior to bregma encompassing the core of the lesioned area (rostrocaudal levels, $-1.4,-1.5,-1.6,-1.7$, and $-1.8 \mathrm{~mm}$ from bregma). Injury to the CA1 subfield was evaluated in the medial and intermediate portion of the CA1 under a light microscope at a 100x magnification by manually counting [38]. We defined degenerating neurons as cells with pyknotic, shrunken nuclei and reduced size. Surviving neurons (Nissl-positive and no sign of neurodegeneration) were counted in five fields and expressed as the average number. For the detection of neuronal apoptosis, we used the Apop-Tag Fluorescein in situ Apoptosis Detection Kit (Chemicon International) according to the manufacturer's instructions [39]. Apoptotic neurons (caspase 3-positive neurons) were counted in five different sections [40]. Cell counting was done with blind assessments by two evaluators.

\section{Blood-brain barrier assay}

For this assay, we used a commercially available BBB kit (MBT-24H, PharmaCo-Cell Co., Nagasaki, Japan), which 
was composed of monkey brain capillary endothelial cells, rat brain pericytes, and rat brain astrocytes. The in vitro $\mathrm{BBB}$ models were established within $4 \mathrm{~d}$ of seeding the cells according to the manufacturer's instructions [41, 42]. Trans-endothelial electrical resistance (TEER), which primarily reflects the flux of sodium ions through the cell layers in the culture conditions, was measured with an epithelial-volt-ohm meter and an Endohm-6 chamber electrode (World Precision Instruments, Sarasota, FL, USA) [42, 43]. The TEERs of the coated, cell-free filters were subtracted from the measured TEER values of the models and shown as $\Omega \mathrm{cm}^{2}$. The apparent permeability constants (Papp) between the luminal and abluminal chambers were calculated based on the distribution ratios $[42,44]$. The expression of endothelial RAGE was silenced using RAGE small hairpin RNA (shRNA) and the pSilencer 3.0-H1 vector (Ambion, Austin, TX) with Lipofectamine 2000 (Thermo Fisher Scientific) [42, 45]. The RAGE-knockdown efficiency was $>90 \%$ with our shRNA system (data not shown).
A

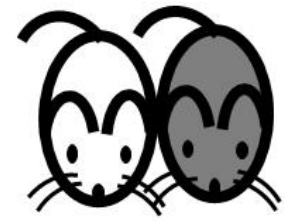

$\mathbf{W T} \rightarrow \underline{W T}$

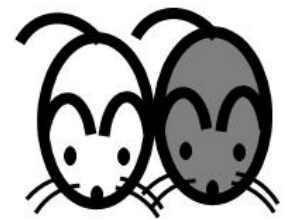

$\mathrm{Tg} \rightarrow \underline{\mathrm{WT}}$

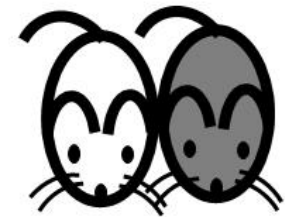

$\mathrm{Tg} \rightarrow \underline{\mathrm{KO}}$

B

Experimental timeline

$$
\text { Day } 0
$$

7

14
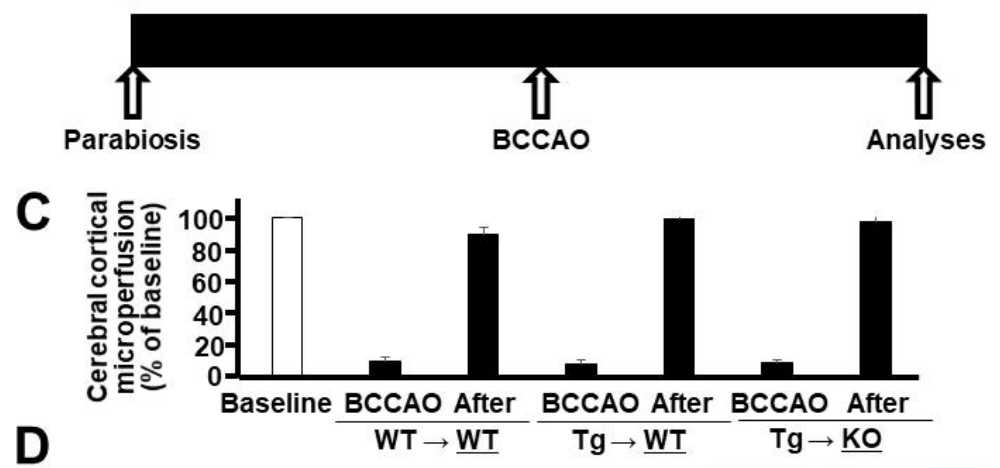

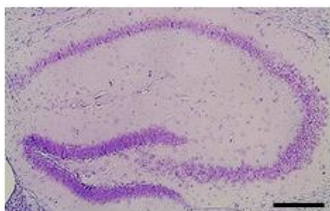

$\mathrm{WT} \rightarrow \underline{\mathrm{WT}}$

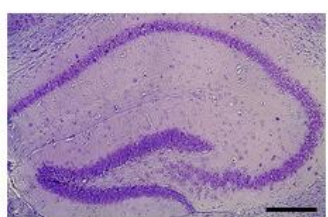

$\mathrm{Tg} \rightarrow \underline{\mathrm{WT}}$

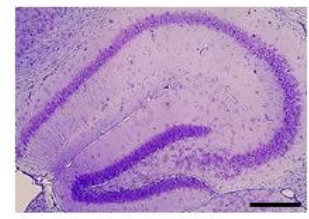

$\mathrm{Tg} \rightarrow \underline{\mathrm{KO}}$

$\mathbf{E}$

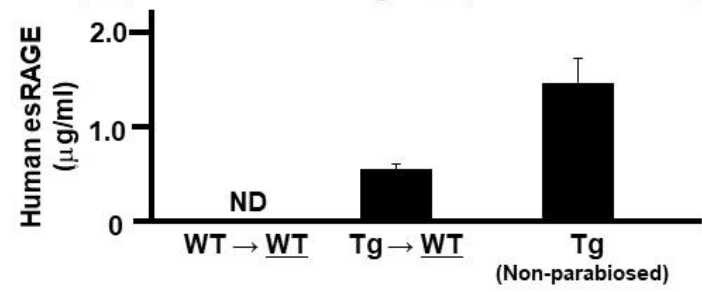

Figure 1. Parabiosis and BCCAO. A) Parabiosis was done between wild-type $(\mathrm{WT})$ and WT mice $(\mathrm{WT} \rightarrow \underline{\mathrm{WT}})$, esRAGE transgenic and WT mice $(\mathrm{Tg} \rightarrow \underline{\mathrm{WT}})$, and esRAGE transgenic and $\overline{R A G E ~ k n o c k o u t ~}(\mathrm{KO})$ mice $(\mathrm{Tg} \rightarrow \underline{\mathrm{KO}})$; gray-colored and right-side mice underwent BCCAO and further analyses. B) Experimental timeline. C, Laser-Doppler flowmetry data for evaluating the cerebral cortical microperfusion. Baseline, baseline data (100\%); BCCAO, data at 1 min during the occlusion; After, data at $30 \mathrm{~min}$ after BCCAO. Values are mean $\pm \mathrm{SD}$. D) Hematoxylin-eosin stain of the hippocampus. E) Human esRAGE levels in the sera of WT sides of WT $\rightarrow \underline{\mathrm{WT}}$ and $\mathrm{Tg} \rightarrow \underline{\mathrm{WT}}$ pairs and non-parabiosed esRAGE Tg mice $(n=4-8)$. ND, not detected. Values are mean \pm SEM. 


\section{Statistical analysis}

$P$ values were calculated using two-tailed Student's t-test for pair wise comparisons, and one-way analysis of variance (ANOVA), followed by Bonferroni's test for multiple comparisons, unless otherwise stated. A P value of $<0.05$ was considered statistically significant. These analyses were carried out with the use of Ekuseru-Toukei 2015 (Social Survey Research Information Co., Ltd., Japan).

\section{RESULTS}

\section{Blood esRAGE protects against neuronal cell damage}

To elucidate the functional role of blood circulating esRAGE, we employed mouse parabiosis approaches by using human esRAGE Tg (human esRAGE overexpressed in blood), RAGE KO, and WT mice (Fig. 1A): (1) WT mice parabiosed with WT mice (WT $\rightarrow$ WT); (2) esRAGE $\mathrm{Tg}$ mice parabiosed with WT mice $(\mathrm{Tg} \rightarrow \mathrm{WT})$; (3) esRAGE Tg mice parabiosed with RAGE$\mathrm{KO}$ mice $(\mathrm{Tg} \rightarrow \mathrm{KO})$. By using these three parabiosis pairs, brain ischemia was induced on either side of the pair (gray-colored right one) to investigate the neuronal cell damage and survival $7 \mathrm{~d}$ after BCCAO (Fig. 1B). LaserDoppler flowmetry data showed no differences in ischemic conditions during and after BCCAO among the three groups (Fig. 1C). Severe ischemic brain damages were not observed after BCCAO (Fig. 1D). The transfer of human esRAGE protein from the esRAGE-Tg mice to the blood of WT mice in the $\mathrm{Tg} \rightarrow \underline{\mathrm{WT}}$ pair was confirmed prior to the experiment. The results indicated that the level of human esRAGE in WT mice was the half of that of esRAGE $\mathrm{Tg}$ mice in the $\mathrm{Tg} \rightarrow \mathrm{WT}$ pair (Fig. 1E).
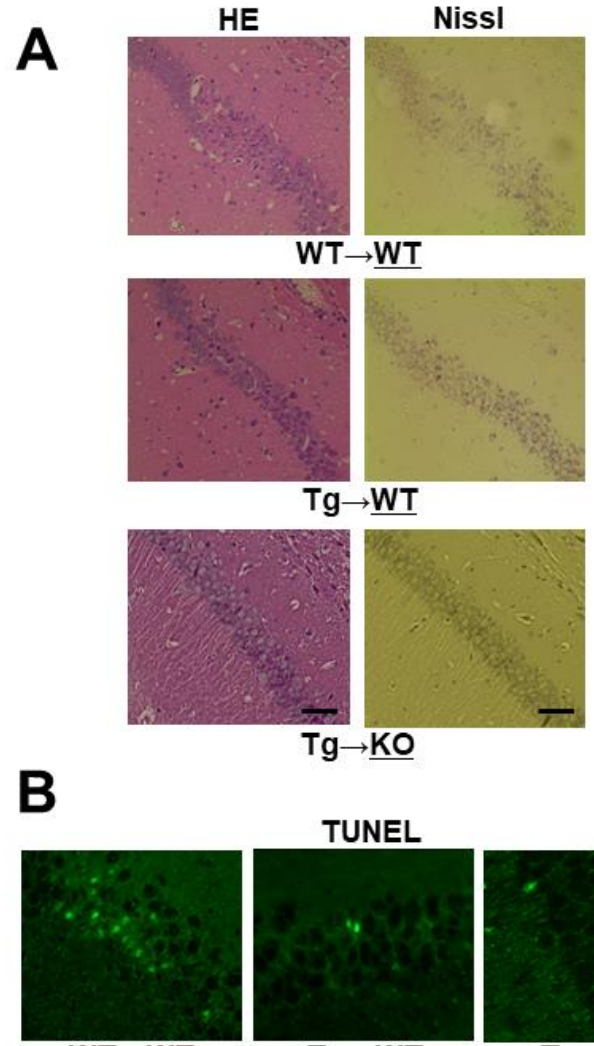

$\mathrm{WT} \rightarrow \underline{\mathrm{WT}}$
$\mathbf{W T} \rightarrow \underline{\mathrm{WT}}$

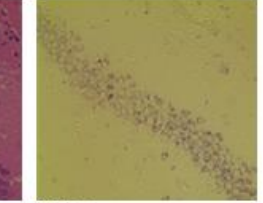

$\mathrm{Tg} \rightarrow \underline{\mathrm{WT}}$

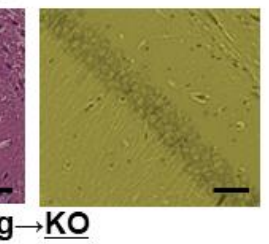

$\mathrm{Tg} \rightarrow \underline{\mathrm{KO}}$

TUNEL

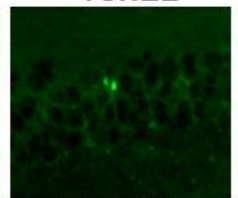

$\mathrm{Tg} \rightarrow \underline{\mathrm{WT}}$

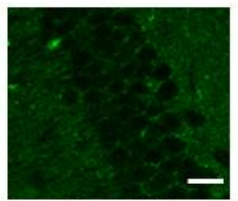

$\mathrm{Tg} \rightarrow \underline{\mathrm{KO}}$
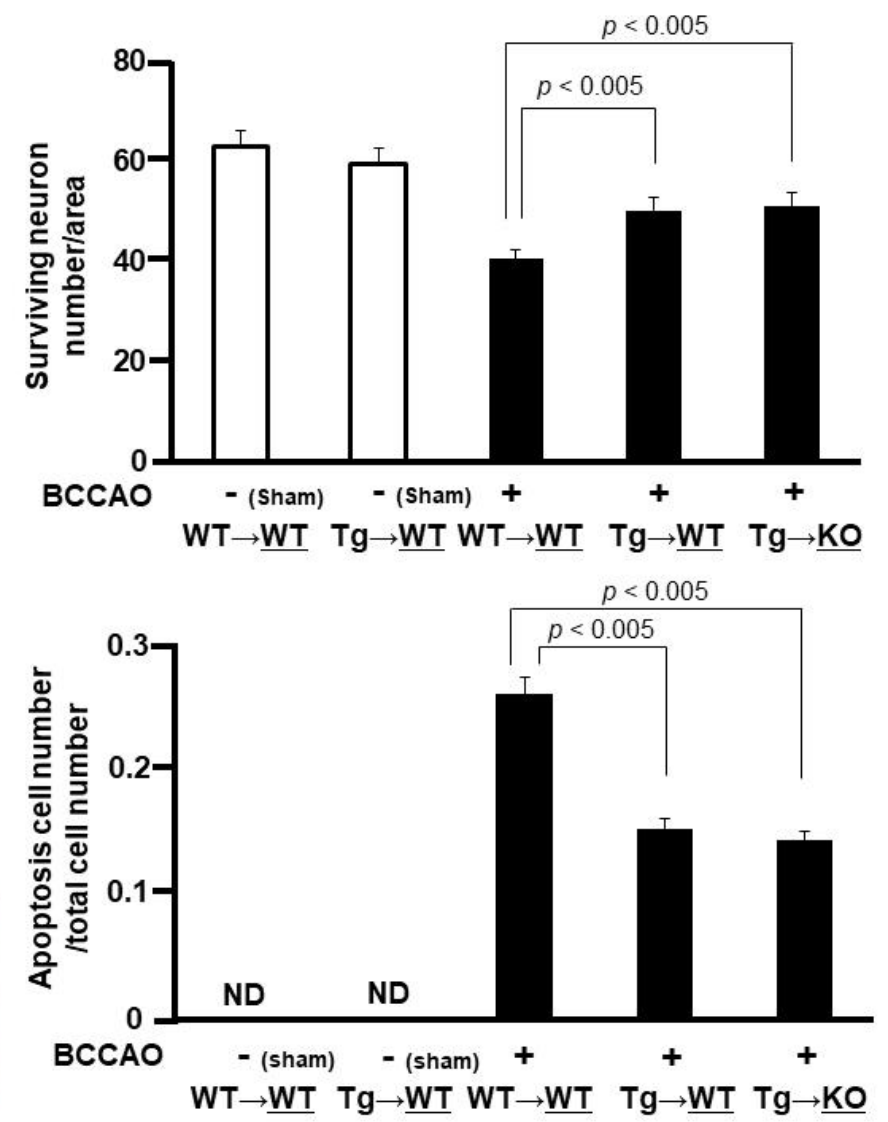

Figure 2. Neuronal cell damage. A) HE and Nissl stains of the hippocampal CA1 region of WT sides of WT $\rightarrow \underline{W T}$ and Tg $\rightarrow \underline{W T}$ pairs and of $\mathrm{KO}$ side of $\mathrm{Tg} \rightarrow \underline{\mathrm{KO}}$ pair after 7 days of BCCAO (left panel). Surviving neuron numbers per area in hippocampal CA1 region were counted in WT sides of WT $\rightarrow \underline{\mathrm{WT}}$ and $\mathrm{Tg} \rightarrow \underline{\mathrm{WT}}$ pairs and in $\mathrm{KO}$ side of $\mathrm{Tg} \rightarrow \underline{\mathrm{KO}}$ pair with or without BCCAO (right panel) $(\mathrm{n}=4-8)$. Values are mean \pm SEM. B) TUNEL stain. Green signals indicate apoptotic cells of WT sides of WT $\rightarrow$ WT and $\mathrm{Tg} \rightarrow \underline{\mathrm{WT}}$ pairs and of KO side of $\mathrm{Tg} \rightarrow \underline{\mathrm{KO}}$ pair after 7 days of BCCAO (left panel). Apoptosis cell numbers per total cell numbers were counted in WT sides of WT $\rightarrow$ WT and $\mathrm{Tg} \rightarrow \mathrm{WT}$ pairs and in $\mathrm{KO}$ side of $\mathrm{Tg} \rightarrow \underline{\mathrm{KO}}$ pair with or without BCCAO (right panel) (n $=4-8$ ). Values are mean \pm SEM. 
Subsequently, we investigated the delayed neuronal cell death and cell survival by using a mouse BCCAO model. Both the hematoxylin-eosin and Nissl stainings demonstrated severe neurodegeneration in the CA1 neurons in the WT side of the WT $\rightarrow \underline{\text { WT }}$ pair, but not in the WT side of the Tg-WT pair, $7 \mathrm{~d}$ after BCCAO (Fig. 2A). Quantitative analyses also showed a significant decrease in the number of delayed neuronal cell death in the CA1 region of the WT side of the $\mathrm{Tg} \rightarrow \underline{\mathrm{WT}}$ pair, when compared with that of the WT $\rightarrow$ WT pair (Fig. 2A). The terminal deoxynucleotidyl transferase dUTP nick end labeling staining was performed to evaluate apoptosis and demonstrated a significantly decreased number of apoptotic cells in the CA1 region of the WT side of the $\mathrm{Tg} \rightarrow$ WT pair, in comparison with the WT $\rightarrow$ WT pair (Fig. 2B). These results suggested neuroprotective effects of esRAGE. However, we could not find any further synergistic or addictive neuroprotective effects of esRAGE under RAGE-deleted conditions; e.g. the KO side of the $\mathrm{Tg} \rightarrow \underline{\mathrm{KO}}$ parabiosis pair (Fig. $2 \mathrm{~A}$ and $\mathrm{B}$ ). We speculate that the neuroprotective effects observed in the $\mathrm{KO}$ side of the $\mathrm{Tg} \rightarrow \underline{\mathrm{KO}}$ parabiosis pair could be caused by only RAGE-deletion.
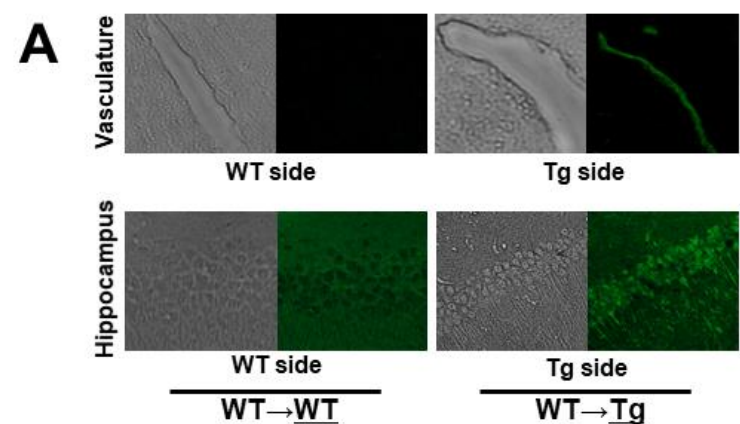

B

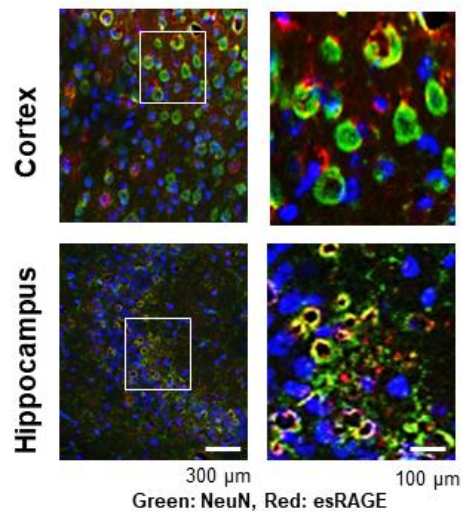

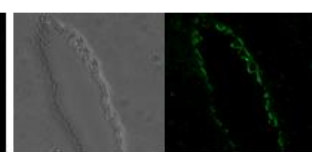

WT side
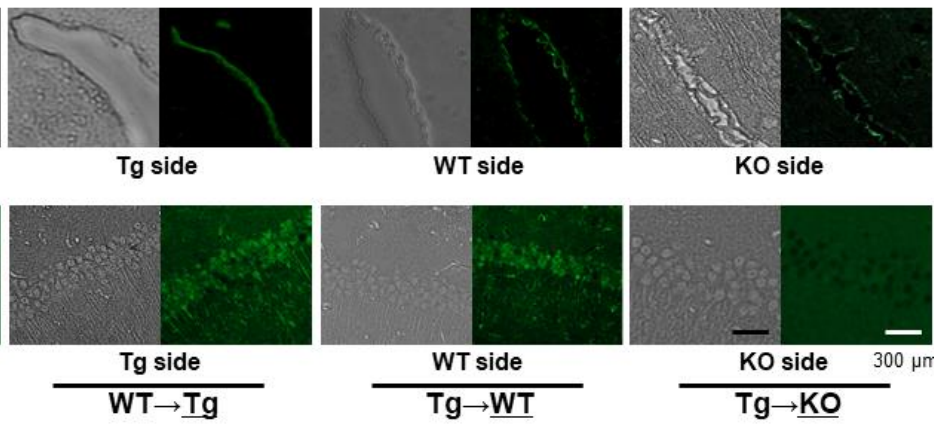

KO side
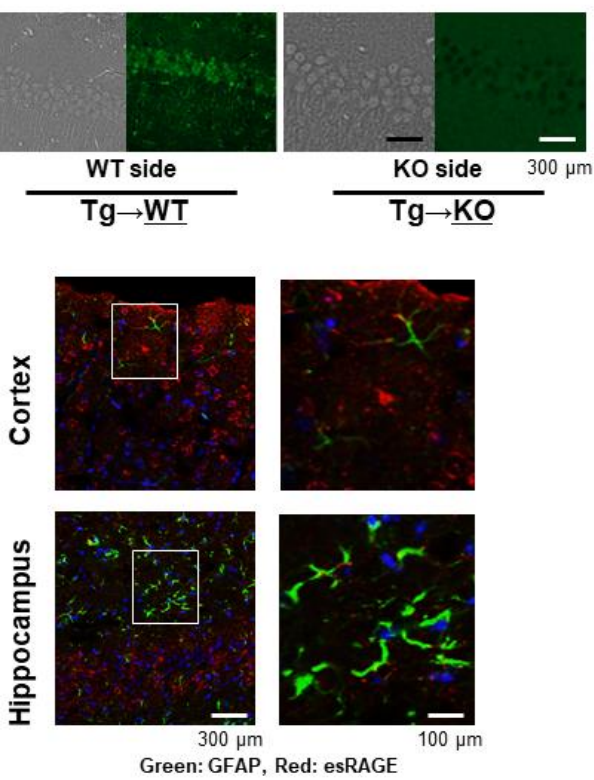

Figure 3. Immunohistochemical detection of human esRAGE. A) Immunohistochemical study for the detection of human esRAGE (green signals). Hippocampal vasculatures and CA1 regions of WT side of WT $\rightarrow \underline{\text { WT }}$ and esRAGE Tg $(\mathrm{Tg}) \rightarrow \underline{\text { WT }}$ pairs, the Tg side of the WT $\rightarrow \underline{\mathrm{Tg}}$ pair, and the RAGE knockout $(\mathrm{KO})$ side of the $\mathrm{Tg} \rightarrow \underline{\mathrm{KO}}$ pair without BCCAO. B) Immunostaining for NeuN (a neuronal marker, green) and human esRAGE (red) (left panel) as well as GFAP (a glial marker, green) and human esRAGE (red) (right panel) in brain cortex and hippocampus of WT sides of Tg $\rightarrow$ WT pair. Blue signals indicate nuclei [4',6-diamidino-2-phenylindole (DAPI) stain].

\section{Endothelial RAGE transfers blood circulating esRAGE to the brain}

To address how endothelial esRAGE can protect against neuronal cell death in the ischemic brain, we first checked the in vivo presence of the human esRAGE protein by immunofluorescent examinations by using mouse parabiosis models. Therefore, we employed WT $\rightarrow \underline{\mathrm{WT}}$, $\mathrm{WT} \rightarrow \underline{\mathrm{Tg}}, \mathrm{Tg} \rightarrow \underline{\mathrm{WT}}$, and $\mathrm{Tg} \rightarrow \underline{\mathrm{KO}}$ parabiosis pairs $\underline{\text { and }}$ examined the underlined animals in each pair after 7 days of the parabiosis. Based on the results, human esRAGE signals were detected on the brain endothelial surface in the WT $\rightarrow \underline{\mathrm{Tg}}, \mathrm{Tg} \rightarrow \underline{\mathrm{WT}}$, and $\mathrm{Tg} \rightarrow \underline{\mathrm{KO}}$ pairs, but not in the $\mathrm{WT} \rightarrow \underline{\mathrm{WT}}$ pair (Fig. 3A). Surprisingly, the human esRAGE protein was also observed in the brain parenchyma around the hippocampal CA1 neurons in the $\mathrm{WT} \rightarrow \underline{\mathrm{Tg}}$ and $\mathrm{Tg} \rightarrow \underline{\mathrm{WT}}$ parabiosis pairs (Fig. 3A). A costaining experiment also showed that the esRAGE signals 
were co-localized with NeuN, a neuronal marker, but not GFAP, an astrocytic maker (Fig. 3B). Based on these results, we suggested that the blood circulating esRAGE and endothelium-associated esRAGE proteins were transferred into the brain parenchyma through the BBB. No positive signals of human esRAGE protein were observed in the $\mathrm{KO}$ side of $\mathrm{Tg} \rightarrow \underline{\mathrm{KO}}$ or $\mathrm{WT} \rightarrow \underline{\mathrm{KO}}$ pair which was a negative control (Fig. 3A). In addition, a quantitative evaluation with ELISA demonstrated that human esRAGE was detectable and its concentration was significantly lower in the $\mathrm{KO}$ side of the brain parenchyma in $\mathrm{Tg} \rightarrow \underline{\mathrm{KO}}$ when compared with the WT side of $\mathrm{Tg} \rightarrow \underline{\mathrm{WT}}$ pair (Fig. 4A). These findings also support our hypothesis that esRAGE transfers into brain through the BBB. The transfer of esRAGE might depend on RAGE, even though some detectable level of human esRAGE was observed in the $\mathrm{KO}$ side of $\mathrm{Tg} \rightarrow \underline{\mathrm{KO}}$ pair (Fig. 4A), which may be derived from the contamination of vessel esRAGE. This is based on the fact that serum levels of human esRAGE were $2.0-3.0 \mu \mathrm{g} / \mathrm{ml}$ in the $\mathrm{Tg}$ side of $\underline{\mathrm{Tg}} \rightarrow \mathrm{WT}$ and $\mathrm{Tg} \rightarrow \mathrm{KO}$ pairs and $\sim 1.0 \mu \mathrm{g} / \mathrm{ml}$ in the WT or $\mathrm{KO}$ side of $\mathrm{Tg} \rightarrow$ WT or $\mathrm{Tg} \rightarrow \underline{\mathrm{KO}}$ pairs (Fig. 4B). Mouse sRAGE levels were found to be $\sim 8 \mathrm{ng} / \mathrm{ml}$ in WT mice, and at under detectable levels in the KO mice (Fig. 4C), indicating that mouse sRAGE is more than two orders of magnitude lower than human esRAGE levels in the serum of Tg parabiosed mice.

B

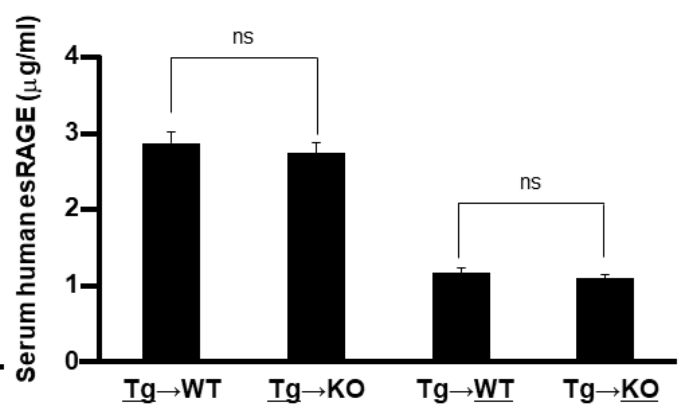

C

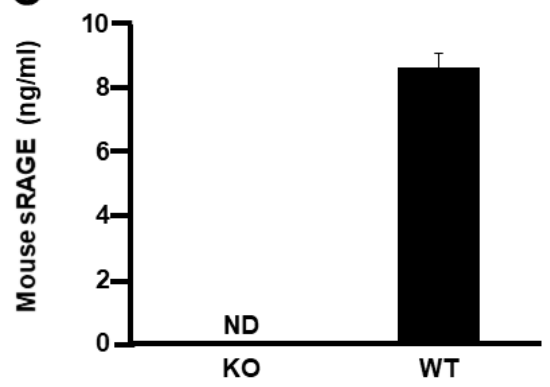

Figure 4. Quantitative detection of human esRAGE A) Human esRAGE contents in the brain parenchyma of WT side of WT $\rightarrow \underline{\text { WT }}$ pair, WT side of $\mathrm{Tg} \rightarrow \underline{\mathrm{WT}}$ pair, $\mathrm{KO}$ side of $\mathrm{Tg} \rightarrow \underline{\mathrm{KO}}$ pair, and Tg side of $\mathrm{WT} \rightarrow \underline{\mathrm{Tg}}$ pair (n =3). B) Serum human esRAGE concentrations in $\mathrm{Tg}$ side of $\mathrm{Tg} \rightarrow \mathrm{WT}$ pair, $\mathrm{Tg}$ side of $\mathrm{Tg} \rightarrow \mathrm{KO}$ pair, WT side of $\mathrm{Tg} \rightarrow \underline{\mathrm{WT}}$ pair, and $\mathrm{KO}$ side of $\mathrm{Tg} \rightarrow \underline{\mathrm{KO}}$ pair $(\mathrm{n}=3)$. C, Serum mouse sRAGE concentrations in KO and WT mice $(\mathrm{n}=3)$. ND, not detected; ns, not significant. Values are mean $\pm \mathrm{SEM}$.

\section{Association of esRAGE with heparan sulfate proteoglycan (HSPG) of vascular endothelial cells}

We further investigated whether there was an association between esRAGE and the cell surface of human HBEC in cultures by using fluorescence microscopy. Faint signals of esRAGE were detected in the endothelial cells, which became much more enhanced with the addition of recombinant esRAGE into the cell culture media (Fig. $5 \mathrm{~A})$. On the other hand, treatment with heparin $(0.1$
$\mathrm{IU} / \mathrm{ml})$ or heparitinase $(1 \mathrm{mU} / \mathrm{ml})$ prevented the accumulation of esRAGE with or without the addition of esRAGE protein in HBEC (Fig. 5A). We obtained the same data in human HMVEC (data not shown). Colocalization of esRAGE and heparin sulfate signals were observed (Fig. 5B). Subsequently, we examined the release of esRAGE protein into the cell culture media of human HBEC with or without treatment with heparin and heparitinase. Based on the results, treatment with heparin $(0.1 \mathrm{IU} / \mathrm{ml})$ significantly increased the concentration of 
esRAGE protein in the cell culture media of HBEC (Fig. 5C). Pretreatment of the HBEC culture media with recombinant esRAGE $(1 \mu \mathrm{g} / \mathrm{ml})$ for $1 \mathrm{~h}$ increased the basal level of esRAGE. Similarly, heparin $(0.1 \mathrm{IU} / \mathrm{ml})$ or heparitinase $(1 \mathrm{mU} / \mathrm{ml})$ also significantly upregulated the esRAGE concentration (Fig. 5C). Furthermore, intravenous heparin injection significantly increased the serum concentration of human esRAGE in vivo in the esRAGE Tg mice (Fig. 5D). These results indicate that esRAGE is associated with HSPG of the endothelial cells.

In order to confirm our hypothesis of the esRAGE transfer through the BBB, we used an in vitro BBB model composed of brain endothelial cells, pericytes, and astrocytes, whose barrier function was intact with higher TEER levels (Fig. 6A). When $20 \mu \mathrm{g} / \mathrm{ml}$ of human esRAGE protein was added to the vessel side, the esRAGE concentrations of the brain side increased significantly in a time-dependent manner without endothelial RAGE knockdown (control) (Fig. 6B). In contrast, no such increase was observed in the RAGE knockdown model (Fig. 6B), indicating that the transfer of human esRAGE protein occurred through the endothelial RAGE.
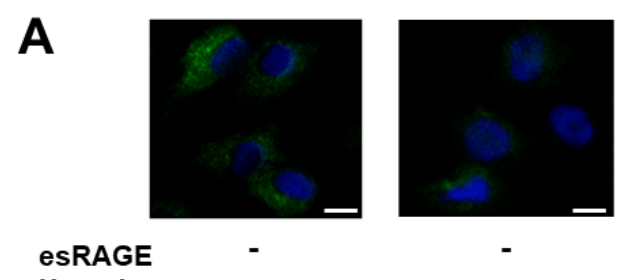
Heparin Heparitinase
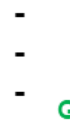

Green, esRAGE Blue, nuclei

B

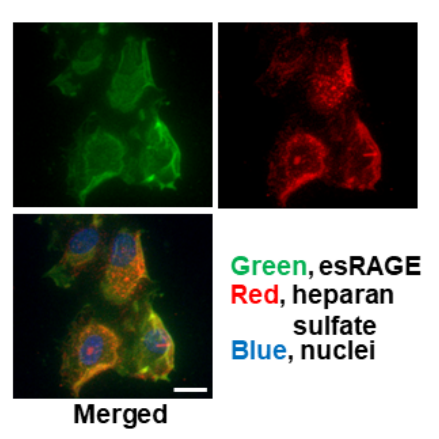

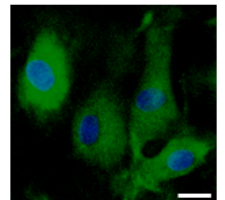

$+$

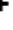

$-$

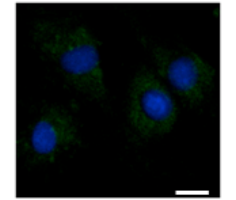

$+$

$+$

$+$

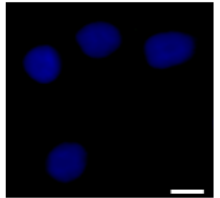

$+$

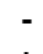

$+$

\section{C}

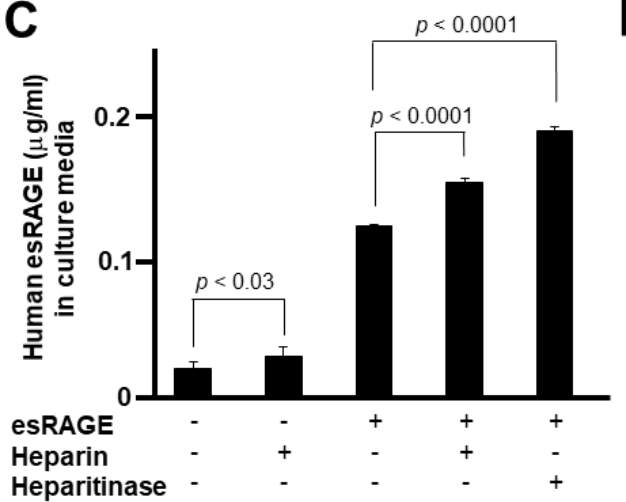

D

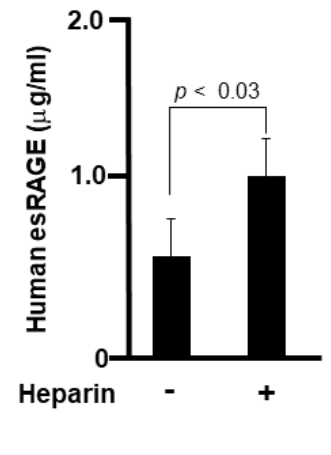

Figure 5. Association of esRAGE with endothelial cells. A and B) Immunofluorescence studies of human brain endothelial cells (HBEC) in culture. Green, Red and blue signals indicate esRAGE, heparin sulfate and nucleus (DAPI), respectively. Bar, $50 \mu \mathrm{m}$. C) Human esRAGE levels in culture media of HBEC $(\mathrm{n}=4)$. esRAGE, 1 $\mu \mathrm{g} / \mathrm{ml}$; Heparin, $0.1 \mathrm{IU} / \mathrm{ml}$; heparitinase, $1 \mathrm{mU} / \mathrm{ml}$. Values are mean \pm SEM. C) Serum levels of human esRAGE in the esRAGE Tg mice with or without heparin injection $(n=4-8)$. Values are mean \pm SEM.

\section{DISCUSSION}

Previous reports demonstrated a pivotal role of neuronal and microglia RAGE in ischemia-induced neuronal death and inflammation in mouse models of distal permanent middle cerebral artery occlusion (MCAO), which cause focal ischemic stroke [46, 47]. On the other hand, in the BCCAO model, which is known to induce global ischemia and subsequent delayed neuronal cell death, the induction of RAGE expression in the hippocampal vascular endothelial cells preceded that neurons and glia, indicating that endothelial RAGE could contribute to delayed neuronal death by enhancing vascular injuries, and potentially leading to microcirculatory disturbances [16]. For the therapeutic strategies against stroke and neuronal cell death, both esRAGE and enzymatically cleaved soluble RAGE were applied to ischemic brain injury models of MCAO and BCCAO. Administration of soluble RAGE has been reported to reduce the infarct size of the brain and microglia/macrophage infiltrations in a mouse MCAO model [48]. Using esRAGE-Tg mice, delayed neuronal cell death in the hippocampus was attenuated in the BCCAO model [16]. However, the precise mechanisms of the decoy-type receptor of soluble RAGE and esRAGE in the protection against ischemiainduced neural damage are still unclear. 


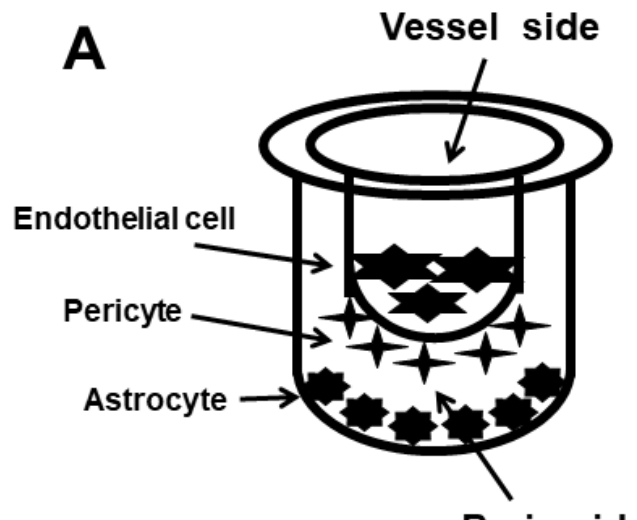

B
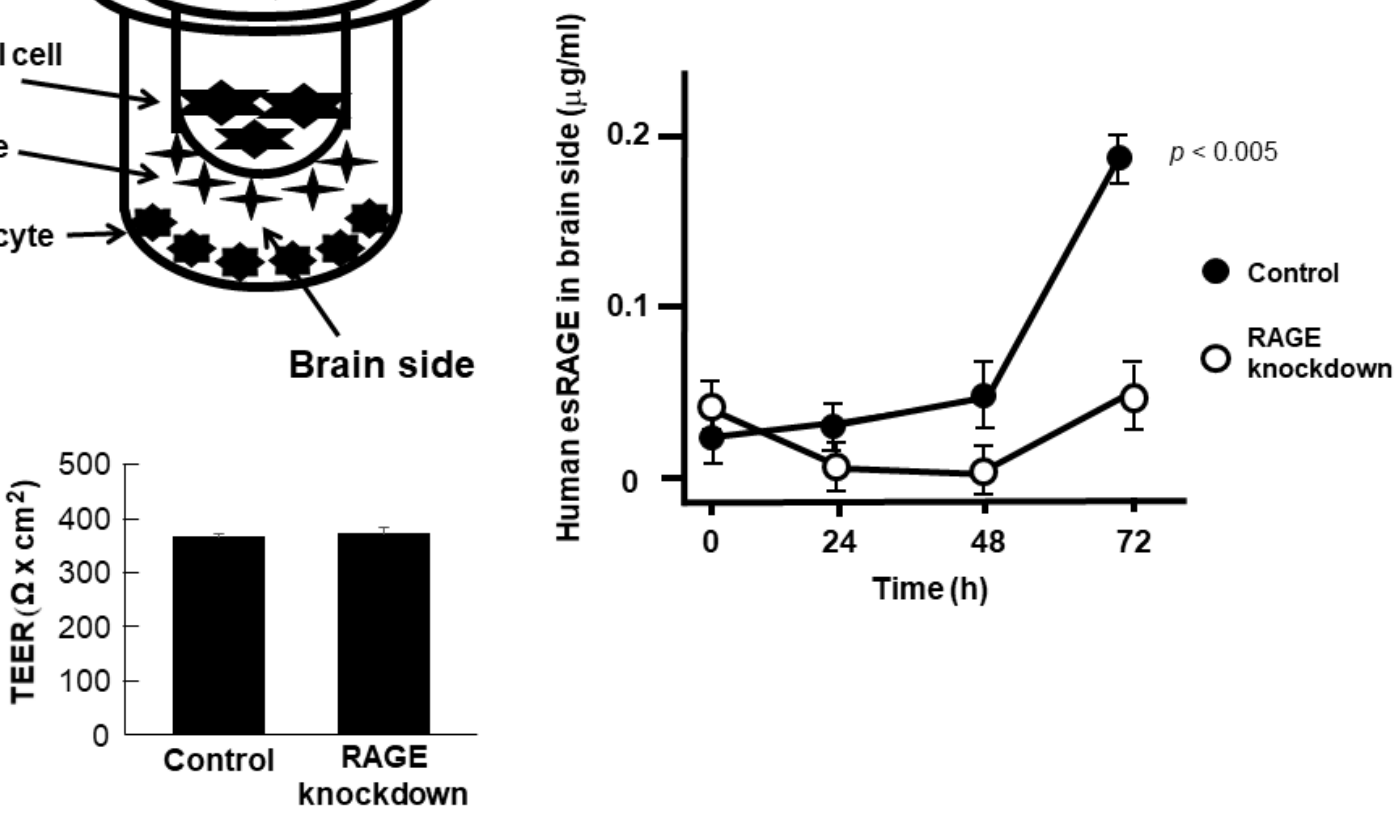

Figure 6. Transfer of esRAGE through BBB. A) In vitro (BBB) model system composed of primary cultures of monkey brain capillary endothelial cells coupled with rat pericytes and astrocytes. Recombinant esRAGE ( $20 \mu \mathrm{g} / \mathrm{ml})$ was added to the upper (vessel side) chambers of the model and transferred esRAGE level was quantified in the lower (brain side) chambers. Endothelial cells were treated with scrambled (control) or RAGE shRNA vectors (knockdown). The integrity of the in vitro primate BBB was unaffected by RAGE knockdown, assessed by high trans-endothelial electrical resistance (TEER) $(n=5)$. B) Human esRAGE levels in brain side were quantified $(\mathrm{n}=5)$. Control, scrambled vector-treated; RAGE knockdown, RAGE shRNA vectors-treated. Values are mean \pm SEM.

In this study, we first observed that esRAGE was associated with HSPG of the vascular endothelial cell surface in which it accumulated both in vitro and in vivo (Fig. 1), which is consistent with previous findings [49]. We also found that excessive circulating esRAGE was protective against delayed neuronal damage in ischemia in our parabiosis models (Fig. 2). Therefore, this study demonstrated for the first time that esRAGE was transferred into the brain from blood through the BBB and localized around the hippocampal CA1 neurons (Fig. 3). These results suggested a functional role of the decoy type receptor against RAGE ligands including damageassociated molecular patterns released in brain ischemia. The human esRAGE is approximately $50 \mathrm{kDa}$ (of a large molecular size) and is usually considered to be untransportable through the BBB. However, using $\mathrm{Tg} \rightarrow \mathrm{WT}$ and $\mathrm{Tg} \rightarrow \mathrm{KO}$ parabiosis mice in addition to the in vitro $\mathrm{BBB}$ model with or without RAGE knockdown, we found that endothelial RAGE could function as a transporter of esRAGE protein from blood to brain (Figs. 3-6). We speculated that RAGE may form an oligomer complex with esRAGE on endothelial cells, which has been previously described whereby RAGE itself forms oligomer with its extracellular domain and transcytoses esRAGE from the luminal to the abluminal side [50]. These findings suggested that both circulating and endothelium-associated and accumulated esRAGE could protect against vascular damages in ischemia; similarly, the esRAGE transferred into the brain could also function as a neuroprotective factor in ischemic brain injuries. Further studies are required to explore how esRAGE can prevent neuronal damage in brain ischemia. It is conceivable that endothelial RAGE is a double-edged sword in brain ischemia; intracellular RAGE signal transduction can induce vascular inflammation and result in the derangement of microvascular circulation, while RAGE can transport the neuroprotective esRAGE into the brain and prevent neuronal cell death. Thus, a condition enriched with esRAGE could be neuroprotective in ischemia.

In conclusion, we confirmed that esRAGE could prevent neuronal cell death in the BCCAO ischemic 
mouse model and could be transferred into the brain through the $\mathrm{BBB}$, where it accumulates around the neurons and functions as a neuroprotective factor. Accordingly, we suggest that a supplementation of esRAGE in ischemic brains will be a potential therapeutic tool for the prevention of neuronal cell death.

\section{Acknowledgements}

We thank Ms Yuko Niimura for her assistance. Participated in research design: YS and YY designed the research. YS, AH, SM, MO, TH, OH, YK, and NL performed the research. YS, HY, MN, YY, and YH analyzed the data. YS, YY, and YH wrote the article. This work was supported by Grants-in-aid for Scientific Research from the Japan Society for the Promotion of Science (grant \#26861143 to YS, grant \# 17K16634 to MO and grant \# 18K06889 to YY), the Program for Fostering Globally Talented Researchers from Japan Society for Promotion of Sciences, and Kanazawa University SAKIGAKE project 2018.

\section{References}

[1] Moskowitz MA, Lo EH, Iadecola C, et al. (2010). The science of stroke: mechanisms in search of treatments. Neuron, 29: 181-98.

[2] Prabhakaran S, Ruff I and Bernstein RA (2015). Acute stroke intervention: A systematic review. JAMA, 313: 1451-1462.

[3] Pulsinelli WA, Levy DE, Duffy TE, et al. (1982). Regional cerebral blood flow and glucose metabolism following transient forebrain ischemia. Ann Neurol, 11: 499-502.

[4] Tajiri S, Oyadomari S, Yano S, et al. (2004). Ischemia-induced neuronal cell death is mediated by the endoplasmic reticulum stress pathway involving CHOP. Cell Death and Differentiation, 11: 403-415.

[5] Kirino T (1982). Delayed neuronal death in the gerbil hippocampus following ischemia. Brain Res, 6: 57-69.

[6] Wang WP, Iyo AH, Miguel-Hidalgo J, et al. (2006). Agmatine protects against cell damage induced by NMDA and glutamate in cultured hippocampal neurons. Brain Res, 21: 210-216.

[7] Ma L1, Carter RJ, Morton AJ, et al. (2003). RAGE is expressed in pyramidal cells of the hippocampus following moderate hypoxic-ischemic brain injury in rats. Brain Res, 966: 167-174.

[8] Koyama R, Tao K, Sasaki T, et al. (2012). GABAergic excitation after febrile seizures induces ectopic granule cells and adult epilepsy. Nat Med, 18: 1271-1278.
Lein ES, Zhao X, Gage FH, et al. (2004). Defining a molecular atlas of the hippocampus using DNA microarrays and high-throughput in situ hybridization. J Neurosci, 24: 3879-3889.

[10] Quillinan N, Grewal H, Deng G, et al. (2015). Region-specific role for GluN2B-containing NMDA receptors in injury to Purkinje cells and CA1 neurons following global cerebral ischemia. Neuroscience, 284: 555-565.

[11] Dihné M, Grommes C, Lutzenburg $\mathrm{M}$, et al. (2002). Different mechanisms of secondary neuronal damage in thalamic nuclei after focal cerebral ischemia in rats. Stroke, 33: 3006-3011.

[12] Siesjö BK, Bengtsson F (1989). Calcium fluxes, calcium antagonists, and calcium-related pathology in brain ischemia, hypoglycemia, and spreading depression: a unifying hypothesis. J Cereb Blood Flow Metab, 9: 127-140.

[13] Chen YJ, Nguyen HM, Maezawa I, et al. (2016). The potassium channel $\mathrm{kca} 3.1$ constitutes a pharmacological target for neuroinflammation associated with ischemia/reperfusion stroke. J Cereb Blood Flow Metab, 36: 2146-2161.

[14] Love S (1999). Oxidative stress in brain ischemia. Brain Pathol, 9: 119-131.

[15] Kumar R, Bukowski MJ, Wider JM, et al. (2016). Mitochondrial dynamics following global cerebral ischemia. Mol Cell Neurosci, 76: 68-75.

[16] Kamide T, Kitao Y, Takeichi T, et al. (2012). RAGE mediates vascular injury and inflammation after global cerebral ischemia. Neurochem Int, 60: 220-228.

[17] Sajjad Muhammad, Waleed Barakat, Stoyan Stoyanov, et al. (2008). The HMGB1 Receptor RAGE Mediates Ischemic Brain Damage. J Neurosci, 28: 12023-12031.

[18] Qiu J, Nishimura M, Wang Y, et al. (2008). Early release of HMGB-1 from neurons after the onset of brain ischemia. J Cereb Blood Flow Metab, 28:927-938.

[19] Kim Y, Kim C, Son SM, et al. (2016). The novel RAGE interactor PRAK is associated with autophagy signaling in Alzheimer's disease pathogenesis. Mol Neurodegener, 11: 4.

[20] Myint KM, Yamamoto Y, Doi T, et al. (2006). RAGE control of diabetic nephropathy in a mouse model: effects of RAGE gene disruption and administration of low-molecular weight heparin. Diabetes, 55: 2510-2522.

[21] Bucciarelli LG, Wendt T, Qu W, et al. (2002) RAGE blockade stabilizes established atherosclerosis in diabetic apolipoprotein E-null mice. Circulation, 106: 2827-2835. 
[22] Yamamoto Y, Yamamoto H (2012). Controlling the receptor for advanced glycation end-products to conquer diabetic vascular complications. J Diabetes Investig, 3: 107-114.

[23] Yan SD, Chen X, Fu J, et al. (1996). RAGE and amyloid-beta peptide neurotoxicity in Alzheimer's disease. Nature, 382: 685-691.

[24] Yamamoto Y, Kato I, Doi T, et al. (2001). Development and prevention of advanced diabetic nephropathy in RAGE-overexpressing mice. J Clin Invest, 108: 261-268.

[25] Tanaka N, Yonekura H, Yamagishi S, et al. (2000). The receptor for advanced glycation end products is induced by the glycation products themselves and tumor necrosis factor- $\alpha$ through nuclear factor- $\kappa \mathrm{B}$, and by $17 \beta$-estradiol through Sp-1 in human vascular endothelial cells. J Biol Chem, 275: 25781-25790.

[26] Yonekura H, Yamamoto Y, Sakurai S, et al. (2003). Novel splice variants of the receptor for advanced glycation end-products expressed in human vascular endothelial cells and pericytes, and their putative roles in diabetes-induced vascular injury. Biochem J, 370: 1097-1109.

[27] Yamagishi S, Matsui T (2010). Soluble form of a receptor for advanced glycation end products (sRAGE) as a biomarker. Front Biosci (Elite Ed), 2: 1184-1195.

[28] Sugihara T, Munesue S, Yamamoto Y et al. (2012). Endogenous secretory receptor for advanced glycation end-products inhibits amyloid- $\beta 1-42$ uptake into mouse brain. J Alzheimers Dis, 28: 709-720.

[29] Yamamoto Y, Doi T, Kato I, et al. (2005). Receptor for advanced glycation end products is a promising target of diabetic nephropathy. Ann N Y Acad Sci, 1043: 562-566.

[30] Muhammad S, Barakat W, Stoyanov S, et al. (2008). The HMGB1 receptor RAGE mediates ischemic brain damage. J Neurosci, 28: 1202312031.

[31] Hassid BG, Nair MN, Ducruet AF, et al. (2009). Neuronal RAGE expression modulates severity of injury following transient focal cerebral ischemia. J Clin Neurosci, 16: 302-306.

[32] Nakatsuji H, Kishida K, Sekimoto R, et al. (2014). Tracing the movement of adiponectin in a parabiosis model of wild-type and adiponectinknockout mice. FEBS Open Bio, 4: 276-282.

[33] Sakurai S, Yamamoto Y, Tamei H, et al. (2006). Development of an ELISA for esRAGE and its application to type 1 diabetic patients. Diabetes Res Clin Pract, 73(2): 158-165.
[34] Takeuchi A, Yamamoto Y, Tsuneyama K, et al. (2007). Endogenous secretory receptor for advanced glycation endproducts as a novel prognostic marker in chondrosarcoma. Cancer, 109(12): 2532-2540.

[35] Cheng C, Tsuneyama K, Kominami R, et al. (2005). Expression profiling of endogenous secretory receptor for advanced glycation end products in human organs. Mod Pathol, 18(10): 1385-1396.

[36] Labat-Moleur F, Guillermet C, Lorimier P, et al. (1998). TUNEL apoptotic cell detection in tissue sections: critical evaluation and improvement. J Histochem Cytochem, 46: 327-334.

[37] Shimamura M, Zhou P, Casolla B, et al. (2013). Prostaglandin E2 type 1 receptors contribute to neuronal apoptosis after transient forebrain ischemia. J Cereb Blood Flow Metab, 33: 12071214.

[38] Kurinami H, Shimamura M, Ma T, et al. (2014). Prohibitin viral gene transfer protects hippocampal CA1 neurons from ischemia and ameliorates postischemic hippocampal dysfunction. Stroke, 45: 1131-1138.

[39] Sasaki T, Kitagawa K, Omura-Matsuoka E, et al. (2007). The phosphodiesterase inhibitor rolipram promotes survival of newborn hippocampal neurons after ischemia. Stroke, 38: 1597-1605.

[40] Cheng Y, Deshmukh M, D'Costa A, et al. (1998). Caspase inhibitor affords neuroprotection with delayed administration in a rat model of neonatal hypoxic-ischemic brain injury. J Clin Invest, 101: 1992-1999.

[41] Bohara M, Kambe Y, Nagayama T, et al. (2014). C-type natriuretic peptide modulates permeability of the blood-brain barrier. J Cereb Blood Flow Metab, 34: 589-596.

[42] Yamamoto Y, Mingkun Liang, Seiichi Munesue, et al. (2019). Vascular RAGE transports oxytocin into the brain to elicit its maternal bonding behaviour in mice. Commun Biol, 2: 76.

[43] Srinivasan B, Kolli AR, Esch MB, et al. (2015). TEER measurement techniques for in vitro barrier model systems. J Lab Autom, 20: 107-126.

[44] Gardner TW, Lieth E, Khin SA, et al. (1997). Astrocytes increase barrier properties and ZO-1 expression in retinal vascular endothelial cells. Invest Ophthalmol Vis Sci, 38: 2423-2427.

[45] Monden M, Koyama H, Otsuka Y, et al. (2013). Receptor for advanced glycation end products regulates adipocyte hypertrophy and insulin sensitivity in mice: involvement of Toll-like receptor 2. Diabetes, 62: 478-489. 
[46] Shichita T, Sugiyama Y, Ooboshi H, et al. (2009). Pivotal role of cerebral interleukin-17-producing gammadeltaT cells in the delayed phase of ischemic brain injury. Nat Med, 15: 946-950.

[47] Shichita T, Hasegawa E, Kimura A, et al. (2012). Peroxiredoxin family proteins are key initiators of post-ischemic inflammation in the brain. Nat Med, 18: 911-917.

[48] Tang SC, Yeh SJ, Tsai LK, et al. (2016). Cleaved but not endogenous secretory RAGE is associated with outcome in acute ischemic stroke. Neurology, 86: 270-276.
[49] Mizumoto S, Takahashi J, Sugahara K, et al. (2012). Receptor for advanced glycation end products (RAGE) functions as receptor for specific sulfated glycosaminoglycans, and antiRAGE antibody or sulfated glycosaminoglycans delivered in vivo inhibit pulmonary metastasis of tumor cells. J Biol Chem, 287: 18985-18994.

[50] Deane R, Du Yan S, Submamaryan RK, et al. (2003). RAGE mediates amyloid-beta peptide transport across the blood-brain barrier and accumulation in brain. Nat Med, 9: 907-913. 